\title{
Comprehensive Echocardiographic and Speckle Tracking Strain Analysis in Rheumatic Mitral Stenosis Patients before and after Transvenous Mitral Commissurotomy
}

Aslannif Bin Roslan ( $\sim$ elysess1@gmail.com )

Institut Jantung Negara

Faten A Aris

IJN: INSTITUT JANTUNG NEGARA

Tey Yee Sin

IJN: INSTITUT JANTUNG NEGARA

Afif Ashari

IJN: INSTITUT JANTUNG NEGARA

Abdul A Shaparudin

IJN: INSTITUT JANTUNG NEGARA

Wan Faizal W Rahimi Shah

IJN: INSTITUT JANTUNG NEGARA

Lee Tjen Jhung

IJN: INSTITUT JANTUNG NEGARA

Koh Hui Beng

IJN: INSTITUT JANTUNG NEGARA

Ahmad Tantawi Jauhari Aktifanus

IJN: INSTITUT JANTUNG NEGARA

Amin Ariff Nuruddin

INSTITUT JANTUNG NEGARA

\section{Research Article}

Keywords: Echocardiography, rheumatic mitral valve, mitral stenosis, strain

Posted Date: November 9th, 2021

DOI: https://doi.org/10.21203/rs.3.rs-1041308/v1

License: (9) This work is licensed under a Creative Commons Attribution 4.0 International License.

Read Full License 
Version of Record: A version of this preprint was published at The International Journal of Cardiovascular Imaging on January 3rd, 2022. See the published version at https://doi.org/10.1007/s10554-021-025183. 


\section{Abstract}

\section{Purpose}

Percutaneous Transvenous Mitral Commissurotomy (PTMC) is the first line treatment for rheumatic mitral stenosis (MS). We sought to evaluate 1) changes in 2-dimensional (2D) echocardiographic and strain values and 2) differences in these values for patients in atrial fibrillation (AF) and sinus rhythm (SR) pre, immediately and 6 months post PTMC.

\section{Methods}

Retrospective study of 136 patients who underwent PTMC between 2011 and 2021. We analyzed their 2D echocardiogram, Global Longitudinal Strain (GLS), Left Atrial Reservoir Strain (LAr-S) and Right Ventricle Free Wall Strain (RVFW-S) pre, immediately and 6 months post PTMC.

Results

At 6 months, mitral valve area increases from $0.94 \pm 0.23 \mathrm{~cm}^{2}$ to $1.50 \pm 0.42 \mathrm{~cm}^{2}$. Ejection fraction (EF) did not change post PTMC (pre; $55.56 \pm 6.62 \%$, immediate; $56.68 \pm 7.83 \%, 6$ months; $56.28 \pm 7.00 \%$, $\mathrm{p}=0.218$ ). Even though EF is preserved, GLS is lower pre-procedure; $-11.52 \pm 3.74 \%$ with significant improvement at 6 months; $-15.16 \pm 4.28 \%(\mathrm{p}<0.001)$. Tricuspid annular plane systolic excursion (TAPSE) improved at 6 months from $1.95 \pm 0.43$ to $2.11 \pm 0.49$ ( $p=0.004)$. RVFW-S increases at 6 months from $-17.37 \pm 6.03 \%$ to $-19.75 \pm 7.19 \%$ ( $p<0.001)$. LAr-S improved from $11.23 \pm 6.83 \%$ pre PTMC to $16.80 \pm$ $8.82 \%$ at 6 months $(p<0.001)$ post PTMC. Pre-procedure patients with AF have lower strain values (More LV, RV and LA dysfunction) with statistically significant difference for LAr-S $(p<0.001), G L S(p<0.001)$ and RVFW-S $(p<0.001)$ than patients in SR.

\section{Conclusion}

Patients with severe rheumatic MS have subclinical left and right ventricle dysfunction despite preserved EF and relatively normal TAPSE with significant improvement seen at 6 months post PTMC. AF patients have lower baseline strain values than SR patients.

\section{Introduction}

Percutaneous Transvenous Mitral Commissurotomy (PTMC) is an established treatment for severe rheumatic mitral stenosis (MS) since its first publication in 1984 [1]. The aim is to crack the fused commissures thereby increasing the mitral valve area and reducing the mean pressure gradient (mean PG) across the mitral valve [2, 3]. Prior studies have shown that the left ventricle (LV) ejection fraction $(\mathrm{EF})$ is preserved in MS and the EF also does not change significantly immediately post PTMC [2-4]. Speckle tracking strain allows us to look at subclinical left ventricle (LV) and right ventricle (RV) dysfunction by analyzing Global Longitudinal Strain (GLS), Right Ventricle Free Wall Strain (RVFW-S) and left atrial dysfunction by looking at Left Atrial Reservoir Strain (LAr-S) [5-8]. With regards to subclinical 
LV and RV dysfunction, there are prior studies showing reduction in Global Longitudinal Strain (GLS) and Right Ventricle Free Wall Strain (RVFW-S), but the study population is small, and these studies only analyzed pre and immediate post PTMC values [7-14]. To our knowledge, this is the first study that tracks all aspects of strain (GLS, RVFW-S and LAr-S) pre, immediately and 6 months post procedure. Atrial fibrillation (AF) commonly coexists with MS and there is no study thus far looking at baseline strain values and response to PTMC in this group of patients compared to those in sinus rhythm (SR) [15-16].

\section{Materials And Methods}

We looked at medical records of all patients who underwent PTMC at our center from 2011 to 2021.All patients included had mitral valve area $<1.5 \mathrm{~cm}^{2}$. We excluded patients with more than mild mitral regurgitation and those whose echocardiographic images were deemed not suitable for strain analysis. This study has been approved by out institution ethics committee.

\section{Echocardiographic data}

All the patients' echocardiographic images were analyzed using the Synapse Cardiovascular software. We analyzed the images looking at 8 echocardiographic parameters. To evaluate the left heart, we measured biplane Simpson EF and left atrial volume index (LAVI), and to evaluate the mitral valve we measured mitral valve area by planimetry (MVA) in short axis view, and mitral valve area by pressure half time (MVA PHT) in apical 4 chamber view. In patients with atrial fibrillation (AF) we averaged 3 cardiac cycles to acquire measurements. For the right heart, we measured systolic pulmonary artery pressure (sPAP), calculated from maximum tricuspid regurgitation velocity (TR Vmax) and inferior vena cava (IVC) size and collapsibility $5 \mathrm{~mm}$ distal to hepatic vein insertion, tricuspid annular plane systolic excursion (TAPSE) and right ventricle fractional area change (FAC).

\section{Strain analysis}

Only patients with suitable apical 4 chamber (A4C), apical 2 chamber (A2C) and apical 3 chamber (A3C) views were included in the study. The strain was analyzed retrospectively using Tom-Tec software. We analyze global longitudinal strain (GLS), right ventricle free wall strain (RVFW-S) and left atrial reservoir strain (LAr-S). Echocardiography and strain analysis was performed for all enrolled patients pre-PTMC (within one-month pre-procedure), immediately post-procedure (one day post-procedure) and 6 months after the procedure. This is the standard echocardiographic follow up protocol for PTMC patients in our setting (Figure 1a,1b and Figure 2a,2b).

\section{Statistical analysis}

Numerical measurements were expressed as mean \pm standard deviation, whereas categorical data expressed as frequency and proportion. A repeated measures ANOVA was used to compare differences between groups at different times points with a Greehouse-Geisser correction and post hoc analysis of 
Bonferroni correction where applicable. $P$ values $<0.05$ were considered statistically significant. Statistical analysis was performed using SPSS ver. 27.0 (SPSS, Chicago, IL, USA).

\section{Results}

Overall, there were 136 patients with severe rheumatic MS who underwent PTMC during a 10-year period between 2011 to 2021 at our center. There were 113 (83.10\%) female and 23 (16.90\%) male patients. All these patients had no or mild MR. Overall age of intervention was $45.05 \pm 13.34$ years old (female; 45.42 \pm 13.77 years old, male; $43.29 \pm 11.03$ years old). The mean Wilkins score was $6.60 \pm 1.61 .82(60.29 \%)$ patients had SR, and $54(39.71 \%)$ patients had AF pre- PTMC. At the time of writing, all these patients are still alive with $16(11.76 \%)$ of them underwent re-intervention. (Table 1)

Table 1

Demographics and Wilkins Score

\begin{tabular}{|llll|}
\hline & Overall & Female & Male \\
\hline $\mathrm{n}(\%)$ & $136(100.00 \%)$ & $113(83.10 \%)$ & $23(16.90 \%)$ \\
\hline Age (Mean \pm SD) & $45.05 \pm 13.34$ & $45.42 \pm 13.77$ & $43.29 \pm 11.03$ \\
\hline Wilkins Score (Mean \pm SD) & $6.60 \pm 1.61$ & $6.58 \pm 1.63$ & $6.70 \pm 1.58$ \\
\hline
\end{tabular}

Analysing the 2D echocardiographic parameters, MVA by planimetry increased from $0.94 \pm 0.23 \mathrm{~cm}^{2}$ to $1.45 \pm 0.38 \mathrm{~cm}^{2}$ post PTMC $(\mathrm{p}<0.001)$ and to $1.50 \pm 0.42 \mathrm{~cm}^{2}$ at 6 months $(\mathrm{p}<0.001)$. The mean PG went down from $10.79 \pm 4.42 \mathrm{mmHg}$ pre to $5.26 \pm 1.97 \mathrm{mmHg}$ post $(p<0.001)$ and $6.21 \pm 2.53$ at 6 months $(p<0.001)$ while the s-PAP went down from $46.61 \pm 21.80 \mathrm{mmHg}$ pre to $34.98 \pm 14.46 \mathrm{mmHg}$ post and $35.12 \pm 12.45 \mathrm{mmHg}$ at 6 months post PTMC (overall $p<0.001$ ). With regards to left heart parameters, the EF was preserved pre-PTMC and there were no statistically significant changes post PTMC or at 6 months (Pre; $55.56 \pm 6.62 \%$ post; $56.68 \pm 7.83 \% 6$ months; $56.28 \pm 7.00 \%$, Overall $p=0.218$ ). LAVI decreased from $82.05 \pm 32.76 \mathrm{mls} / \mathrm{m}^{2}$ pre to $73.12 \pm 28.47 \mathrm{mls} / \mathrm{m}^{2}$ post $(\mathrm{p}=0.001)$ and $76.41 \pm$ $33.17 \mathrm{mls} / \mathrm{m}^{2}(p=0.110)$ at 6 months post procedure. For the right side of the heart, TAPSE did not improve immediately post PTMC but showed improvement at 6 months. [Pre; $1.95 \pm 0.43$, post; $1.93 \pm$ $0.48(p=1.00) 6$ months; $2.11 \pm 0.49(p=0.004)]$. Conversely FAC improved immediately post PTMC $35 \pm$ $11 \%$ to $40 \pm 9 \%(p<0.001)$ but saw no significant gain at 6 months $[39 \pm 10 \%, p=0.947$ (post vs 6 months)]. (Table 2) 
Table 2

Traditional Echocardiographic Parameters for All Patients Pre, Immediate and 6 months post PTMC

\begin{tabular}{|c|c|c|c|c|c|c|c|}
\hline \multirow[t]{2}{*}{ Characteristics } & \multirow[t]{2}{*}{ Pre } & \multirow{2}{*}{$\begin{array}{l}\text { Post } \\
\text { Immediate }\end{array}$} & \multirow{2}{*}{$\begin{array}{l}\text { Post } 6 \\
\text { months }\end{array}$} & \multirow{2}{*}{$\begin{array}{l}\text { Overall } p \\
\text { value }\end{array}$} & \multicolumn{3}{|c|}{$\mathrm{p}$ value post-hoc analysis } \\
\hline & & & & & $\begin{array}{l}\text { Pre-to- } \\
\text { post }\end{array}$ & $\begin{array}{l}\text { Post-to-6 } \\
\text { month }\end{array}$ & $\begin{array}{l}\text { Pre-to } \\
\text { 6- } \\
\text { month }\end{array}$ \\
\hline $\begin{array}{l}\text { MVA } \\
\text { Planimetry }\end{array}$ & $\begin{array}{l}0.94 \pm \\
0.23\end{array}$ & $\begin{array}{l}1.45 \pm \\
0.38\end{array}$ & $\begin{array}{l}1.50 \pm \\
0.42\end{array}$ & $<0.001$ & $<0.001$ & 0.627 & $<0.001$ \\
\hline MVA PHT & $\begin{array}{l}0.97 \pm \\
0.25\end{array}$ & $\begin{array}{l}1.49 \pm \\
0.40\end{array}$ & $\begin{array}{l}1.50 \pm \\
0.42\end{array}$ & $<0.001$ & $<0.001$ & 1.000 & $<0.001$ \\
\hline Mean PG & $\begin{array}{l}10.79 \pm \\
4.42\end{array}$ & $\begin{array}{l}5.26 \pm \\
1.97\end{array}$ & $\begin{array}{l}6.21 \pm \\
2.53\end{array}$ & $<0.001$ & $<0.001$ & $<0.001$ & $<0.001$ \\
\hline s-PAP & $\begin{array}{l}46.61 \pm \\
21.80\end{array}$ & $\begin{array}{l}34.98 \pm \\
14.46\end{array}$ & $\begin{array}{l}35.12 \pm \\
12.45\end{array}$ & $<0.001$ & $<0.001$ & 1.000 & $<0.001$ \\
\hline RV TAPSE & $\begin{array}{l}1.95 \pm \\
0.43\end{array}$ & $\begin{array}{l}1.93 \pm \\
0.48\end{array}$ & $\begin{array}{l}2.11 \pm \\
0.49\end{array}$ & $<0.001$ & 1.000 & 0.001 & 0.004 \\
\hline EF & $\begin{array}{l}55.56 \pm \\
6.62\end{array}$ & $\begin{array}{l}56.68 \pm \\
7.83\end{array}$ & $\begin{array}{l}56.28 \pm \\
7.00\end{array}$ & 0.218 & & & \\
\hline LAVI & $\begin{array}{l}82.05 \pm \\
32.76\end{array}$ & $\begin{array}{l}73.12 \pm \\
28.47\end{array}$ & $\begin{array}{l}76.41 \pm \\
33.17\end{array}$ & 0.001 & 0.001 & 0.410 & 0.110 \\
\hline RV FAC & $\begin{array}{l}0.35 \pm \\
0.11\end{array}$ & $\begin{array}{l}0.40 \pm \\
0.09\end{array}$ & $\begin{array}{l}0.39 \pm \\
0.10\end{array}$ & $<0.001$ & $<0.001$ & 0.947 & 0.006 \\
\hline
\end{tabular}

Analyzing the speckle tracking strain analysis data, it is of interest to note that while EF and TAPSE were normal prior to PTMC, there were significant subclinical left ventricle and right ventricle dysfunction. GLS was markedly depressed pre PTMC; $-11.52 \pm 3.74 \%$ with no improvement immediately post PTMC; -12.19 $\pm 5.64 \%(p=0.632)$. There were, however, significant improvement at 6 months; $-15.2 \pm 4.3 \%(p<0.001)$ (31.60\% improvement). RVFW-S pre PTMC was also depressed at $-17.37 \pm 6.03 \%$. Like GLS and TAPSE it did not improve immediately post-PTMC; $-17.34 \pm 7.32 \%(p=1.000)$ but improved significantly at 6 months; $-19.75 \pm 7.19 \%$ ( $p=0.011)$. Overall improvement in RVFW-S was $13.70 \%$. Finally, LAr-S was the only strain parameter with significant immediate and 6 months improvement post-PTMC (Pre; $11.23 \pm$ $6.83 \%$ to Post; $13.50 \pm 7.83 \%$ ( $p=0.011) 6$ months; $16.80 \pm 8.82 \%(p<0.001)$ with overall improvement of 49.60\%. (Table 3, Figure 3) 
Table 3

Speckle Tracking Analysis, LA reservoir, LA Conduit, LA Booster, GLS and RVFWS for All Patients Pre, Post Immediate and 6 months post PTMC

\begin{tabular}{|c|c|c|c|c|c|c|c|}
\hline \multirow{2}{*}{$\begin{array}{l}\text { Speckle } \\
\text { Tracking }\end{array}$} & \multirow[t]{2}{*}{ Pre } & \multirow{2}{*}{$\begin{array}{l}\text { Post } \\
\text { Immediate }\end{array}$} & \multirow{2}{*}{$\begin{array}{l}\text { Post } 6 \\
\text { months }\end{array}$} & \multirow{2}{*}{$\begin{array}{l}\text { Overall } p \\
\text { value }\end{array}$} & \multicolumn{3}{|c|}{$\mathrm{p}$ value post-hoc analysis } \\
\hline & & & & & $\begin{array}{l}\text { Pre-to- } \\
\text { post }\end{array}$ & $\begin{array}{l}\text { Post-to-6 } \\
\text { month }\end{array}$ & $\begin{array}{l}\text { Pre-to } \\
\text { 6- } \\
\text { month }\end{array}$ \\
\hline LAr-S & $\begin{array}{l}11.23 \pm \\
6.83\end{array}$ & $\begin{array}{l}13.50 \pm \\
7.83\end{array}$ & $\begin{array}{l}16.80 \pm \\
8.82\end{array}$ & $<0.001$ & 0.011 & $<0.001$ & $<0.001$ \\
\hline LAc-S & $\begin{array}{l}6.58 \pm \\
4.42\end{array}$ & $9.12 \pm 4.99$ & $\begin{array}{l}10.41 \pm \\
4.76\end{array}$ & $<0.001$ & $<0.001$ & 0.061 & $<0.001$ \\
\hline LA booster & $\begin{array}{l}8.08 \pm \\
4.05\end{array}$ & $7.65 \pm 4.47$ & $\begin{array}{l}11.42 \pm \\
5.33\end{array}$ & $<0.001$ & 1.000 & $<0.001$ & 0.002 \\
\hline GLS & $\begin{array}{l}-11.52 \pm \\
3.74\end{array}$ & $\begin{array}{l}-12.19 \pm \\
5.64\end{array}$ & $\begin{array}{l}-15.16 \pm \\
4.28\end{array}$ & $<0.001$ & 0.632 & $<0.001$ & $<0.001$ \\
\hline RVFW-S & $\begin{array}{l}-17.37 \pm \\
6.03\end{array}$ & $\begin{array}{l}-17.34 \pm \\
7.32\end{array}$ & $\begin{array}{l}-19.75 \pm \\
7.19\end{array}$ & 0.003 & 1.000 & 0.005 & 0.011 \\
\hline
\end{tabular}

In terms of traditional echocardiographic parameters, patients with AF had lower TAPSE and lower FAC at all measured points pre and post PTMC compared to patients in SR (TAPSE $p<0.001$ and FAC $p=0.010$ ). AF patients had lower strain values (More LV, RV and LA dysfunction) with statistically significant difference for LAr-S $(p<0.001)$, GLS $(p<0.001)$ and RVFW-S $(p<0.001)$ compared to patients in SR. Pre PTMC GLS for AF vs SR; (-9.56 $\pm 3.34 \%$ vs $-12.86 \pm 3.42 \%)$, pre RVFWS AF vs SR; (-14.46 $\pm 5.40 \%$ vs $-19.37 \pm 5.65)$ and pre-LAr-S AF vs SR $(7.58 \pm 5.30 \%$ vs $13.84 \pm 6.63 \%)$.

In AF patients, GLS increased from $-9.56 \pm 3.34 \%$ pre PTMC to $-13.24 \pm 3.91 \%$ at 6 months $(p<0.001$ Absolute improvement $=38.49 \%)$ compared to $-12.86 \pm 3.42 \%$ to $-16.48 \pm 4.04 \%$ at 6 months $(p<0.001$ Absolute improvement $=28.15 \%)$ for SR patients. Amongst patients with AF, the LAr-S improved from 7.58 $\pm 5.30 \%$ to $10.66 \pm 5.03 \%(p=0.028$ Absolute improvement $=40.63 \%)$, whereas for patients in $S R$, it improved from $13.84 \pm 6.63 \%$ to $21.18 \pm 8.34 \%$ ( $<<0.001$ Absolute improvement= 53.03\%). For RVFW-S, AF patients saw numerical improvement $-14.46 \pm 5.40 \%$ to $-16.38 \pm 5.52 \%$ at 6 months $(p=0.232$ Absolute improvement $=13.28 \%$ ) and for SR patients it improved from $-19.37 \pm 5.65 \%$ to $22.07 \pm 7.33 \%$ at 6 months $(p=0.065$ Absolute improvement $=13.94 \%)$. However, for RVFW-S these changes failed to reach statistical significance (Table 4, Table 5, and Figure 4). 
Table 4

Echocardiographic and strain analysis for patients with atrial fibrillation vs patients with sinus rhythm

\begin{tabular}{|c|c|c|c|}
\hline Characteristics & Sinus Rhythm & Atrial Fibrillation & $p$ value \\
\hline \multicolumn{4}{|l|}{ s-PAP } \\
\hline Pre & $46.50 \pm 19.53$ & $46.72 \pm 24.21$ & \multirow[t]{3}{*}{0.222} \\
\hline Post immediate & $34.07 \pm 14.00$ & $35.92 \pm 15.07$ & \\
\hline Post 6 months & $30.63 \pm 9.05$ & $39.73 \pm 13.85$ & \\
\hline \multicolumn{4}{|l|}{ RV TAPSE } \\
\hline Pre & $2.18 \pm 0.31$ & $1.58 \pm 0.33$ & \multirow[t]{3}{*}{$<0.001$} \\
\hline Post immediate & $2.16 \pm 0.39$ & $1.56 \pm 0.34$ & \\
\hline Post 6 months & $2.34 \pm 0.44$ & $1.75 \pm 0.30$ & \\
\hline \multicolumn{4}{|l|}{ RV FAC } \\
\hline Pre & $0.36 \pm 0.11$ & $0.33 \pm 0.11$ & \multirow[t]{3}{*}{0.010} \\
\hline Post immediate & $0.41 \pm 0.08$ & $0.39 \pm 0.09$ & \\
\hline Post 6 months & $0.40 \pm 0.10$ & $0.37 \pm 0.10$ & \\
\hline \multicolumn{4}{|l|}{ LAr-S } \\
\hline Pre & $13.84 \pm 6.63$ & $7.58 \pm 5.30$ & \multirow[t]{3}{*}{$<0.001$} \\
\hline Post immediate & $16.44 \pm 8.14$ & $9.38 \pm 5.11$ & \\
\hline Post 6 months & $21.18 \pm 8.34$ & $10.66 \pm 5.03$ & \\
\hline \multicolumn{4}{|l|}{ LAc-S } \\
\hline Pre & $5.87 \pm 3.54$ & $7.58 \pm 5.30$ & \multirow[t]{3}{*}{0.732} \\
\hline Post immediate & $9.26 \pm 5.23$ & $8.93 \pm 4.71$ & \\
\hline Post 6 months & $10.68 \pm 5.13$ & $10.04 \pm 4.21$ & \\
\hline \multicolumn{4}{|l|}{ GLS } \\
\hline Pre & $-12.86 \pm 3.42$ & $-9.56 \pm 3.34$ & \multirow[t]{3}{*}{$<0.001$} \\
\hline Post immediate & $-14.14 \pm 3.84$ & $-9.35 \pm 6.61$ & \\
\hline Post 6 months & $-16.48 \pm 4.04$ & $-13.24 \pm 3.91$ & \\
\hline \multicolumn{4}{|l|}{ RVFW-S } \\
\hline Pre & $-19.37 \pm 5.65$ & $-14.46 \pm 5.40$ & $<0.001$ \\
\hline
\end{tabular}




\begin{tabular}{|llll|}
\hline Characteristics & Sinus Rhythm & Atrial Fibrillation & p value \\
\hline Post immediate & $-19.49 \pm 7.52$ & $-14.22 \pm 5.83$ & \\
\cline { 1 - 3 } Post 6 months & $-22.07 \pm 7.33$ & $-16.38 \pm 5.52$ & \\
\hline
\end{tabular}

Table 5

Overall changes, Changes for AF vs SR patients

\begin{tabular}{|c|c|c|c|c|c|c|c|}
\hline $\begin{array}{l}\text { Speckle } \\
\text { Tracking }\end{array}$ & Pre & $\begin{array}{l}\text { Post } \\
\text { Immediate }\end{array}$ & $\begin{array}{l}\text { Post } \\
6 \text { months }\end{array}$ & $\begin{array}{l}\% \\
\text { Difference }\end{array}$ & $\begin{array}{l}\mathrm{p} \\
\text { value }^{1}\end{array}$ & $\begin{array}{l}\text { p } \\
\text { value }^{2}\end{array}$ & $\begin{array}{l}\text { p } \\
\text { value }^{3}\end{array}$ \\
\hline \multicolumn{8}{|l|}{ LAr-S } \\
\hline Overall & $\begin{array}{l}11.23 \pm \\
6.83\end{array}$ & $\begin{array}{l}13.50 \pm \\
7.83\end{array}$ & $\begin{array}{l}16.80 \pm \\
8.82\end{array}$ & $49.60 \%$ & $<0.001$ & & \\
\hline Sinus Rhythm & $\begin{array}{l}13.84 \pm \\
6.63\end{array}$ & $\begin{array}{l}16.44 \pm \\
8.14\end{array}$ & $\begin{array}{l}21.18 \pm \\
8.34\end{array}$ & $53.03 \%$ & $<0.001$ & $<0.001$ & 0.020 \\
\hline $\begin{array}{l}\text { Atrial } \\
\text { Fibrillation }\end{array}$ & $\begin{array}{l}7.58 \pm \\
5.30\end{array}$ & $\begin{array}{l}9.38 \pm \\
5.11\end{array}$ & $\begin{array}{l}10.66 \pm \\
5.03\end{array}$ & $40.63 \%$ & 0.028 & & \\
\hline \multicolumn{8}{|l|}{ GLS } \\
\hline Overall & $\begin{array}{l}-11.52 \pm \\
3.74\end{array}$ & $\begin{array}{l}-12.19 \pm \\
5.64\end{array}$ & $\begin{array}{l}-15.16 \pm \\
4.28\end{array}$ & $31.60 \%$ & $<0.001$ & & \\
\hline Sinus Rhythm & $\begin{array}{l}-12.86 \pm \\
3.42\end{array}$ & $\begin{array}{l}-14.14 \pm \\
3.84\end{array}$ & $\begin{array}{l}-16.48 \pm \\
4.04\end{array}$ & $28.15 \%$ & $<0.001$ & $<0.001$ & 0.211 \\
\hline $\begin{array}{l}\text { Atrial } \\
\text { Fibrillation }\end{array}$ & $\begin{array}{l}-9.56 \pm \\
3.34\end{array}$ & $\begin{array}{l}-9.35 \pm \\
6.61\end{array}$ & $\begin{array}{l}-13.24 \pm \\
3.91\end{array}$ & $38.49 \%$ & $<0.001$ & & \\
\hline \multicolumn{8}{|l|}{ RVFW-S } \\
\hline Overall & $\begin{array}{l}-17.37 \pm \\
6.03\end{array}$ & $\begin{array}{l}-17.34 \pm \\
7.32\end{array}$ & $\begin{array}{l}-19.75 \pm \\
7.19\end{array}$ & $13.70 \%$ & 0.011 & & \\
\hline Sinus Rhythm & $\begin{array}{l}-19.37 \pm \\
5.65\end{array}$ & $\begin{array}{l}-19.49 \pm \\
7.52\end{array}$ & $\begin{array}{l}-22.07 \pm \\
7.33\end{array}$ & $13.94 \%$ & 0.065 & $<0.001$ & 0.889 \\
\hline $\begin{array}{l}\text { Atrial } \\
\text { Fibrillation }\end{array}$ & $\begin{array}{l}-14.46 \pm \\
5.40\end{array}$ & $\begin{array}{l}-14.22 \pm \\
5.83\end{array}$ & $\begin{array}{l}-16.38 \pm \\
5.52\end{array}$ & $13.28 \%$ & 0.232 & & \\
\hline
\end{tabular}

* $p$ values ${ }^{1}$ suggested any statistically significance of value differences at \% difference pre to post 6 months

* $p$ values ${ }^{2}$ suggested any statistically significance of value differences between groups regardless different time points

* $p$ values ${ }^{3}$ suggested any statistically significance of possible interaction of value differences between groups and different time points 
Finally, we evaluated whether there were any significant differences between patients who underwent reintervention $(n=16)$ vs those who did not $(n=120)$. The only significant difference was those who needed intervention had lower EF at all measured timepoints compared to those without re-intervention $(p=0.015)$. There was no difference in GLS, RVFW-S and LAr-S between the 2 groups. (Table 6). 
Table 6

Echocardiogram and strain parameters for patients who needs reintervention vs patients who don't need re-intervention

\begin{tabular}{|c|c|c|c|}
\hline Characteristics & No Re-intervention & Re-intervention & $\mathrm{p}$ value \\
\hline \multicolumn{4}{|l|}{ MVA Planimetry } \\
\hline Pre & $0.92 \pm 0.21$ & $1.14 \pm 0.24$ & \multirow[t]{3}{*}{0.386} \\
\hline Post immediate & $1.45 \pm 0.39$ & $1.44 \pm 0.20$ & \\
\hline Post 6 months & $1.50 \pm 0.41$ & $1.50 \pm 0.51$ & \\
\hline \multicolumn{4}{|l|}{ MVA PHT } \\
\hline Pre & $0.96 \pm 0.25$ & $0.99 \pm 0.25$ & \multirow[t]{3}{*}{0.259} \\
\hline Post immediate & $1.49 \pm 0.40$ & $1.49 \pm 0.42$ & \\
\hline Post 6 months & $1.48 \pm 0.36$ & $1.70 \pm 0.71$ & \\
\hline \multicolumn{4}{|l|}{ Mean PG } \\
\hline Pre & $10.86 \pm 4.43$ & $10.33 \pm 4.56$ & \multirow[t]{3}{*}{0.722} \\
\hline Post immediate & $5.19 \pm 1.91$ & $5.76 \pm 2.41$ & \\
\hline Post 6 months & $6.13 \pm 2.50$ & $6.85 \pm 2.78$ & \\
\hline \multicolumn{4}{|l|}{ s-PAP } \\
\hline Pre & $45.75 \pm 21.16$ & $53.40 \pm 26.96$ & \multirow[t]{3}{*}{0.513} \\
\hline Post immediate & $34.75 \pm 14.76$ & $36.78 \pm 12.56$ & \\
\hline Post 6 months & $35.14 \pm 11.39$ & $34.95 \pm 20.04$ & \\
\hline \multicolumn{4}{|l|}{ RV TAPSE } \\
\hline Pre & $1.93 \pm 0.43$ & $2.07 \pm 0.46$ & \multirow[t]{3}{*}{0.766} \\
\hline Post immediate & $1.93 \pm 0.48$ & $1.92 \pm 0.48$ & \\
\hline Post 6 months & $2.11 \pm 0.48$ & $2.09 \pm 0.53$ & \\
\hline \multicolumn{4}{|l|}{ EF } \\
\hline Pre & $56.06 \pm 6.59$ & $51.92 \pm 5.87$ & \multirow[t]{3}{*}{0.015} \\
\hline Post immediate & $57.37 \pm 7.37$ & $51.58 \pm 9.53$ & \\
\hline Post 6 months & $56.71 \pm 6.99$ & $53.08 \pm 6.40$ & \\
\hline \multicolumn{4}{|l|}{ LAr-S } \\
\hline Pre & $11.27 \pm 6.90$ & $10.95 \pm 6.58$ & 0.966 \\
\hline
\end{tabular}




\begin{tabular}{|c|c|c|c|}
\hline Characteristics & No Re-intervention & Re-intervention & $p$ value \\
\hline Post immediate & $13.46 \pm 7.70$ & $13.76 \pm 9.01$ & \\
\hline Post 6 months & $16.76 \pm 8.71$ & $17.04 \pm 9.99$ & \\
\hline \multicolumn{4}{|l|}{ GLS } \\
\hline Pre & $-11.68 \pm 3.74$ & $-10.37 \pm 3.67$ & \multirow[t]{3}{*}{0.886} \\
\hline Post immediate & $-12.11 \pm 5.71$ & $-12.75 \pm 5.32$ & \\
\hline Post 6 months & $-15.14 \pm 4.26$ & $-15.33 \pm 4.61$ & \\
\hline \multicolumn{4}{|l|}{ RVFW-S } \\
\hline Pre & $-17.26 \pm 5.91$ & $-18.08 \pm 7.03$ & \multirow[t]{3}{*}{0.590} \\
\hline Post immediate & $-17.30 \pm 7.47$ & $-17.55 \pm 6.56$ & \\
\hline Post 6 months & $-19.54 \pm 6.93$ & $-21.15 \pm 8.99$ & \\
\hline
\end{tabular}

\section{Discussion}

The standard first line treatment for severe rheumatic MS is PTMC which has been done all over the world since the first publication of its' success in 1984 on 5 patients [1]. The aim of PTMC is to relief the obstruction across the mitral valve by cracking open the fused commissures, hence the name "commissurotomy" [1]. There are many studies showing significant increases in MVA and reduction in mean PG post PTMC [1-3]. PTMC is generally associated with good outcomes with low procedural and long-term mortality, but with significant need for re-intervention in the future. [17-19]

In term of left ventricular function, there is a notion that MS protects the LV, thus systolic function should be preserved [13]. Many studies have shown that the EF is indeed preserved pre and immediately post PTMC [2-4]. Our study further confirms this fact and additionally demonstrates this phenomenon even at 6 months. Strain is a relatively new method to evaluate LV, RV and LA dysfunction before these become apparent using traditional 2D- echocardiographic parameters [20]. The basic principle of strain echocardiography is recognition of 2 points on the myocardium and evaluation of how much these points move away or towards each other during pre-specified periods of the cardiac cycle. This value is expressed as a percentage $[20,21]$. For GLS, the normal value is more negative than $-18 \%$, for RVFW-S normal value is more negative than $-25 \%$ and for LAr-S normal value is more than $45 \%[22,23]$. Initial doppler tissue-based strain showed significant subclinical LV dysfunction in patients with severe MS [5]. With the advent of speckle tracking strain, analysis is easier, with greater reproducibility and not angle dependent [22]. Utilization of speckle tracking strain in previous studies also demonstrated significant reduction in GLS amongst severe MS patients with preserved EF [6-9], with one study demonstrating a relationship between MS progression and GLS [14]. Our study is unique because we track the changes in 
GLS pre, immediately and 6 months post PTMC and found that while GLS did not improve immediately, there was significant improvement 6 months post PTMC with overall improvement of $31.6 \%$.

As a result of the hemodynamic sequelae and back pressure from the obstructed mitral valve, patients with severe rheumatic MS commonly have increase in S-PAP [2, 3]. Despite this, many studies have shown that the TAPSE is still above the cut-off for abnormal values based on the American Society of Echocardiography guidelines (Abnormal TAPSE < 1.7) [22, 23]. In our study the TAPSE pre-PTMC is $1.9 \pm$ 0.4 with no improvement immediately but significantly improve at 6 months (Table 2). With regards to strain, prior to our publication there is a study that showed improvement in RVFS-S immediately post PTMC [8]. Like that study, we also found that RVFW-S was significantly depressed pre-PTMC, and as with GLS and TAPSE there were no immediate improvements, but RVFW-S improved significantly at 6 months post-PTMC.

Patients with severe MS all have left atrial dilatation, and many are at risk of developing atrial fibrillation [15]. In our study the LAr-S was markedly depressed but unlike GLS and RVFW-S, LAr-S improved immediately and continued to improve at 6 months post procedure. The 6 months period required for significant improvement in GLS, and RVFW-S seems to suggest that post PTMC, there is progressive positive remodelling of $L V, R V$, and left atrium.

This study also shows the importance of early intervention before AF develops. All strain parameters are lower for AF patients compared to those in sinus rhythm. Regardless of the rhythm at intervention, both groups of patients had significant improvement in their strain values post PTMC. Finally, there are 16 patients that required re-intervention (4 re-do PTMC and 12 mitral valves replacement). We are unable to find any statistically different strain parameters, except that those who required re-intervention had significantly lower EF.

\section{Conclusions}

Severe Rheumatic MS patients have significant impairment in left ventricle, right ventricle and left atrial function as illustrated by low GLS, RVFW-S and LAr-S. PTMC results in significant improvements in all strain parameters at 6 months post procedure. Patients with AF have significantly reduced strain parameters compared to patients in SR, however they still derive significant improvement from PTMC.

\section{Declarations}

\section{Fundings}

There are no fundings involved in this study

\section{Conflict of interests}

The authors declare that they have no conflict of interest 


\section{References}

1. Inoue K, Owaki T, Nakamura T, Kitamura F, Miyamoto N. Clinical application of transvenous mitral commissurotomy by a new balloon catheter. J Thorac Cardiovasc Surg. 1984 Mar;87(3):394-402.

2. Khan I, Shah B, Habeel Dar M, Khan A, Faisal Iftekhar M, Sami A. Clinical and Echocardiographic Follow-up after Successful Percutaneous Transvenous Mitral Commissurotomy. Cureus. 2017 Sep;9(9):e1726.

3. Khan A, Shafiq I, Jan M, Hassan Z. Echocardiographic assessment before and after Percutaneous Transvenous Mitral Commissurotomy in patients with Rheumatic Mitral Stenosis. Pakistan J Med Sci. 2021;37(1):104-8.

4. Gaasch WH, Folland ED. Left ventricular function in rheumatic mitral stenosis. Eur Heart J [Internet]. 1991 Jul 1;12(suppl_B):66-9. Available from: https://doi.org/10.1093/eurheartj/12.suppl_B.66

5. Dogan S, Aydin M, Gursurer M, Dursun A, Onuk T, Madak H. Prediction of subclinical left ventricular dysfunction with strain rate imaging in patients with mild to moderate rheumatic mitral stenosis. J Am Soc Echocardiogr. 2006 Mar;19(3):243-8.

6. Ozdemir AO, Kaya CT, Ozcan OU, Ozdol C, Candemir B, Turhan S, et al. Prediction of subclinical left ventricular dysfunction with longitudinal two-dimensional strain and strain rate imaging in patients with mitral stenosis. Int J Cardiovasc Imaging [Internet]. 2010;26(4):397-404. Available from: https://doi.org/10.1007/s10554-009-9550-2

7. Simşek Z, Karakelleoğlu S, Gündoğdu F, Aksakal E, Sevimli S, Arslan S, et al. Evaluation of left ventricular function with strain/strain rate imaging in patients with rheumatic mitral stenosis. Anadolu Kardiyol Derg. 2010 Aug;10(4):328-33.

8. Khanna R, Raghuvanshi AS, Kumar S, Garg N, Tewari S, Kapoor A, et al. Immediate impact of percutaneous transvenous mitral commisurotomy on right ventricle longitudinal strain in patients of mitral stenosis. Echocardiography [Internet]. 2018 Oct 1;35(10):1525-32. Available from: https://doi.org/10.1111/echo.14093

9. Bilen E, Kurt M, Tanboga IH, Kaya A, Isik T, Ekinci M, et al. Severity of mitral stenosis and left ventricular mechanics: a speckle tracking study. Cardiology. 2011;119(2):108-15.

10. Caso P, Ancona R, Di salvo G, Pinto S, Macrino M, Palma V, et al. Atrial reservoir function by strain rate imaging in asymptomatic mitral stenosis: Prognostic value at 3 year follow-up. Eur $\mathrm{J}$ Echocardiogr. 2009 Jun 1;10:753-9.

11. Ancona R, Comenale Pinto S, Caso P, Di Salvo G, Severino S, D’Andrea A, et al. Two-dimensional atrial systolic strain imaging predicts atrial fibrillation at 4-year follow-up in asymptomatic rheumatic mitral stenosis. J Am Soc Echocardiogr Off Publ Am Soc Echocardiogr. 2013 Mar;26(3):270-7. 
12. Rohani A, Kargar S, Fazlinejad A, Ghaderi F, Vakili V, Falsoleiman H, et al. Acute effect of treatment of mitral stenosis on left atrium function. Ann Card Anaesth. 2017;20(1):42-4.

13. Sravan K Reddy N, Ranjan Shetty K, Sudhakar Rao M, Sree Madhurya Reddy M. Strain imaging to assess early effects of successful percutaneous balloon mitral valvotomy on left atrium mechanics. Egypt Hear J [Internet]. 2019;71(1):25. Available from: https://doi.org/10.1186/s43044-019-0021-3

14. Gerede DM, Ongun A, Kaya CT, Acıbuca A, Özyüncü N, Erol Ç. Use of strain and strain rate echocardiographic imaging to predict the progression of mitral stenosis: A 5-year follow-up study. Anatol J Cardiol. 2016;16(10):772-7.

15. Pourafkari L, Ghaffari S, Bancroft GR, Tajlil A, Nader ND. Factors associated with atrial fibrillation in rheumatic mitral stenosis. Asian Cardiovasc Thorac Ann. 2015 Jan;23(1):17-23.

16. Westermann D, Schrage B. Mitral stenosis and atrial fibrillation. Vol. 106, Heart (British Cardiac Society). 2020. p. 713.

17. Fazir O Al, Amin A, Robaayah Z, Ali RM. TCT-808 15-year Outcomes and Predictors of Success for Percutaneous Mitral Commissurotomy for Rheumatic Mitral Stenosis. J Am Coll Cardiol [Internet]. 2014 Sep 16;64(11_Supplement):B236-B236. Available from: https://doi.org/10.1016/j.jacc.2014.07.883

18. Murthy Jayanthi Sriram SN, Venkata BJ, Sadagopan T, Ramamurthy MT. Immediate, intermediate and long term clinical outcomes of percutaneous transvenous mitral commissurotomy. IJC Hear Vasc [Internet]. 2015;6:66-70. Available from:

https://www.sciencedirect.com/science/article/pii/S235290671500007X

19. Kim D, Chung H, Nam JH, Park DH, Shim CY, Kim JS, et al. Predictors of long-term outcomes of percutaneous mitral valvuloplasty in patients with rheumatic mitral stenosis. Yonsei Med J. 2018;59(2):273-8.

20. Mondillo S, Galderisi M, Mele D, Cameli M, Lomoriello VS, Zacà V, et al. Speckle-tracking echocardiography: a new technique for assessing myocardial function. $J$ ultrasound Med Off $\mathrm{J}$ Am Inst Ultrasound Med. 2011 Jan;30(1):71-83.

21. Bansal M, Kasliwal RR. How do i do it? Speckle-tracking echocardiography. Indian Heart J [Internet]. 2013;65(1):117-23. Available from: http://dx.doi.org/10.1016/j.ihj.2012.12.004

22. Badano LP, Kolias TJ, Muraru D, Abraham TP, Aurigemma G, Edvardsen T, et al. Standardization of left atrial, right ventricular, and right atrial deformation imaging using two-dimensional speckle tracking echocardiography: a consensus document of the EACVI/ASE/Industry Task Force to standardize deformation imaging. Eur Hear journal Cardiovasc Imaging. 2018 Jun;19(6):591-600.

23. Lang RM, Badano LP, Mor-Avi V, Afilalo J, Armstrong A, Ernande L, et al. Recommendations for cardiac chamber quantification by echocardiography in adults: an update from the American Society of 
Echocardiography and the European Association of Cardiovascular Imaging. J Am Soc Echocardiogr Off Publ Am Soc Echocardiogr. 2015 Jan;28(1):1-39.e14.

\section{Figures}
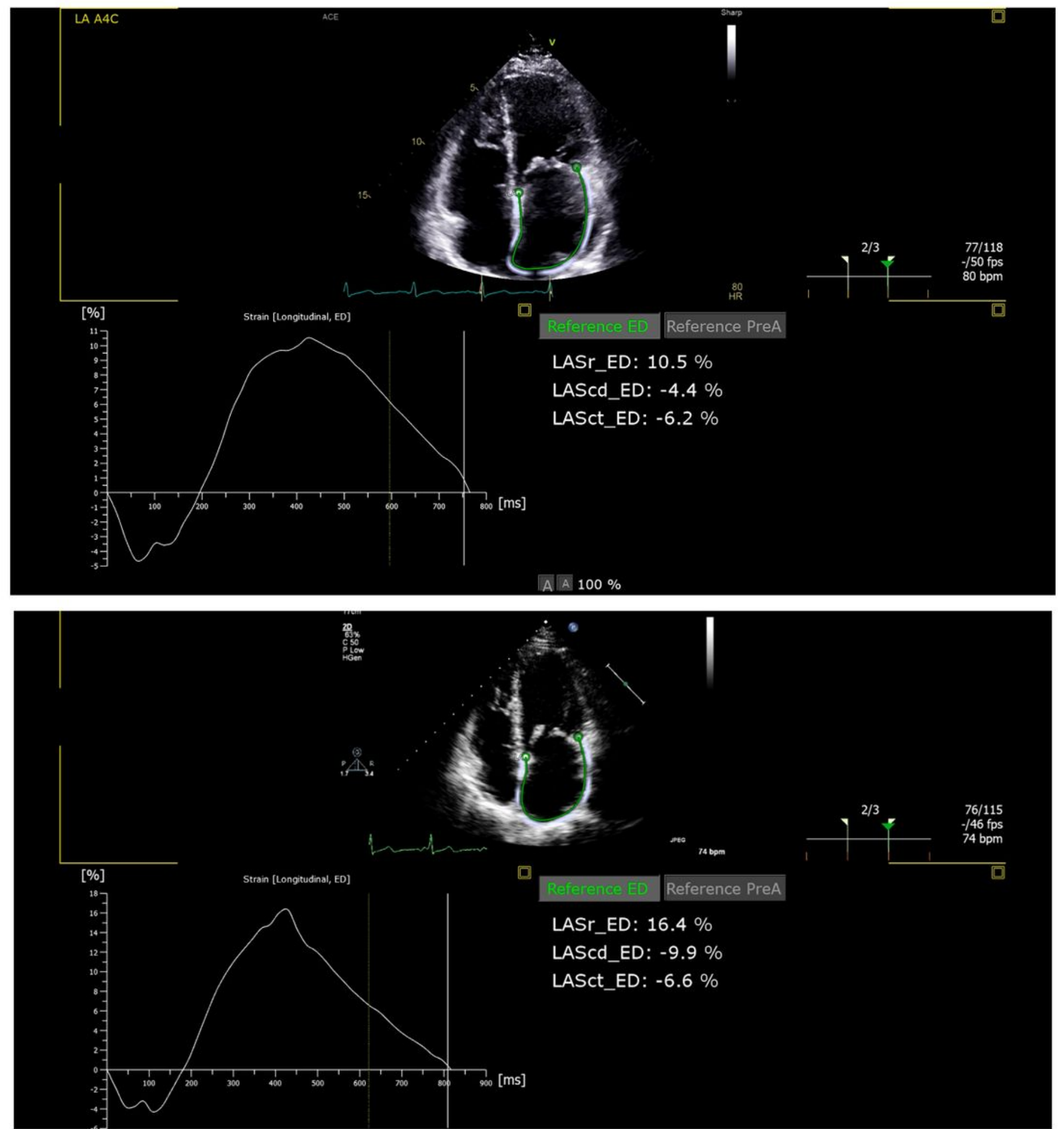

Figure 1 
Example of a patient pre and post strain analysis for left atrial reservoir (Lar-S) and right ventricle free wall (RVFW-S) strain. 1a and 1b Pre-PTMC LA-r S (Figure 1a) of 10.5\% improved to $16.4 \%$ at 6 months post PTMC (Figure 1b)
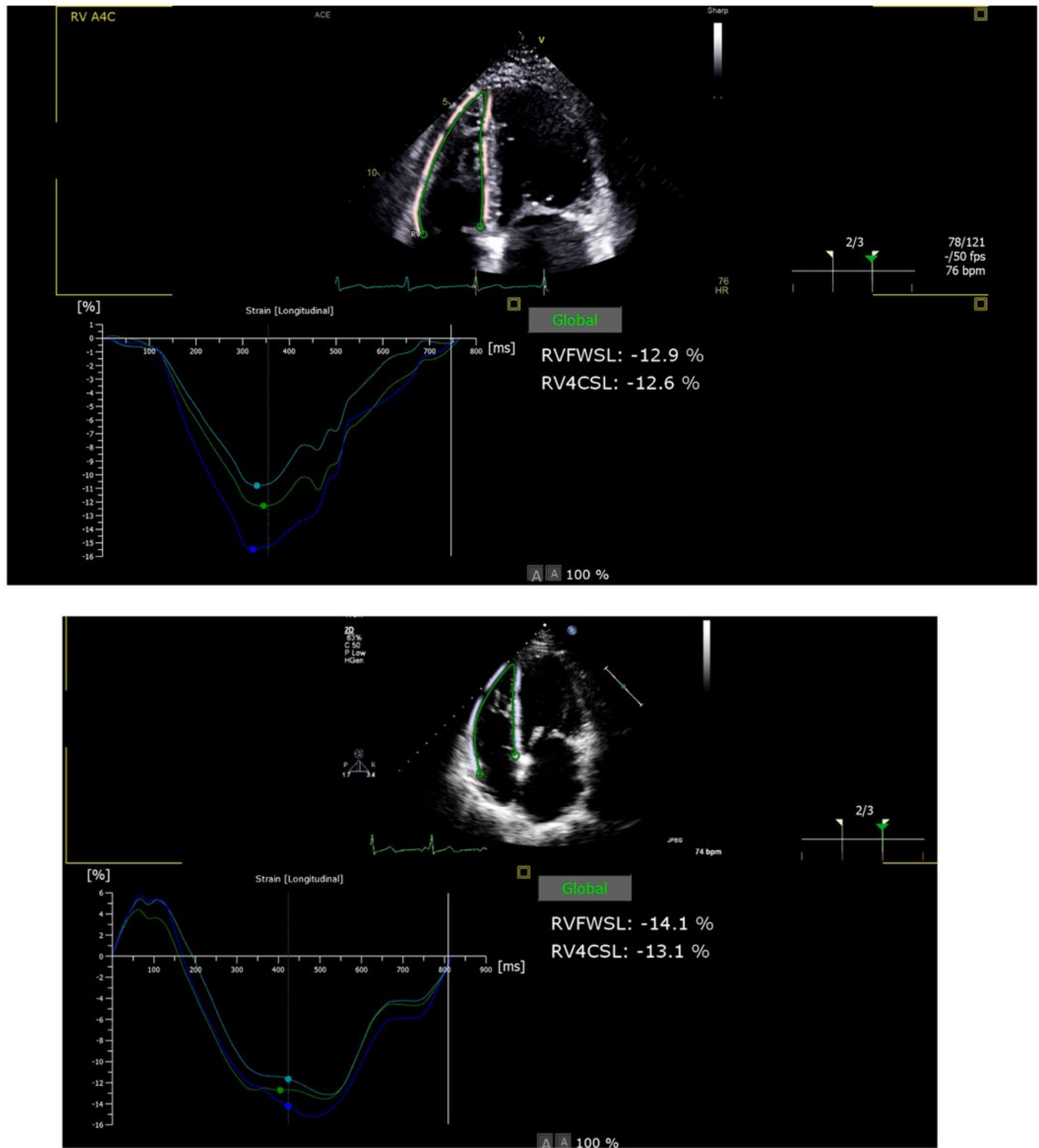

Figure 2

2a and 2b Pre-PTMC RVFW S (Figure $2 a$ ) of $-12.9 \%$ improved to $-14.1 \%$ at 6 months post PTMC (Figure 2b) 


40
30
20
10
0
-10
-20
-30
-40

SPECKLE TRACKING STRAIN PRE, POST AND 6 MONTHS POST PTMC 40

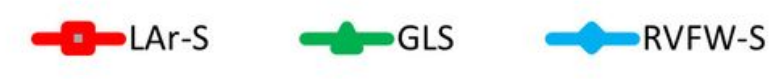

20

10

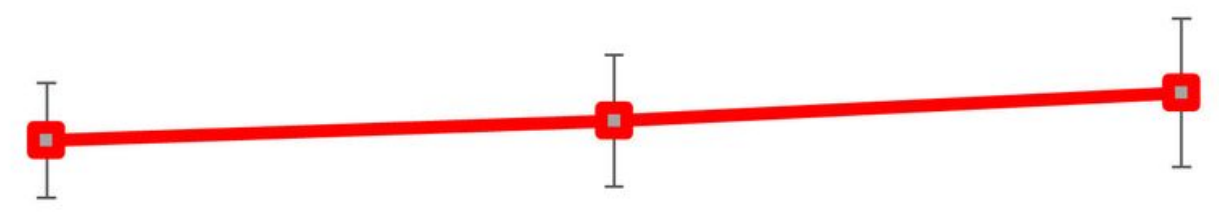

0

$-10$

$-20$

$-30$

$-40$

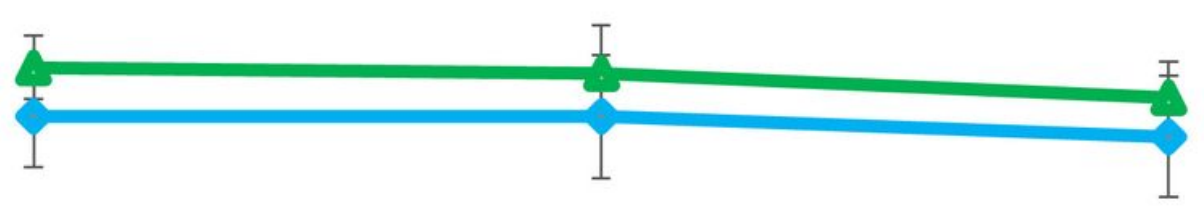

Pre

Post Immediate

Post 6 Months

Figure 3

Strain analysis pre, immediately post and 6 months after PTMC Immediately post procedure, only Left Atrial Reservoir (LAr-S) have statistically significant improvement. However, at 6-months post procedures all 3 strain types improves significantly

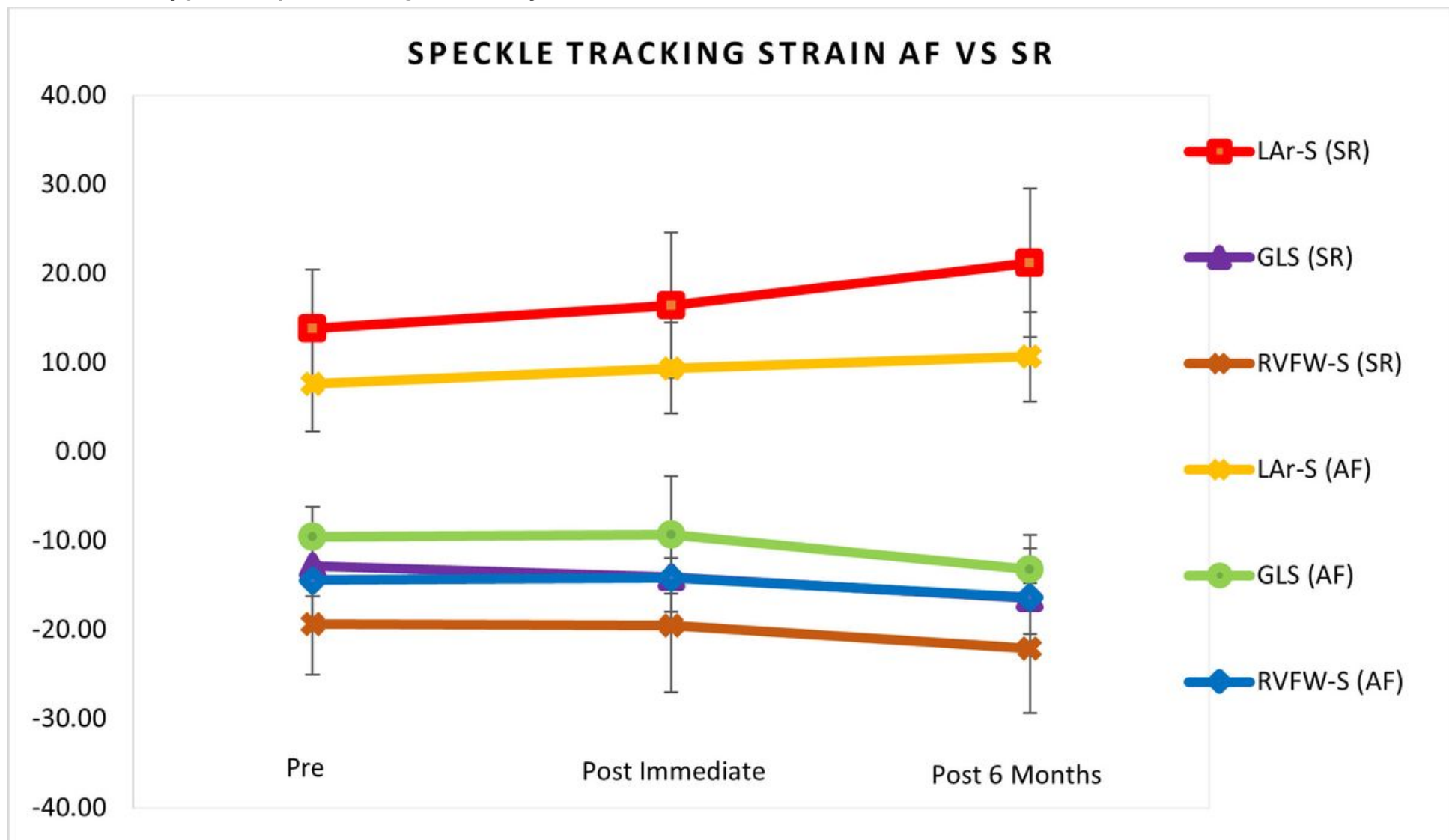

Figure 4 
Strain for patients with AF vs patient in SR Pre-PTMC patients with Atrial Fibrillation (AF) have lower Global Longitudinal Strain (GLS), Right Ventricle Free Wall Strain (RVFW-S) and Left Atrial Reservoir Strain (LAr-S) than patient in Sinus Rhythm (SR) but still derive significant benefit from PTMC 